\title{
A New Improvement of Damping System Analysis Method
}

\author{
Jingfang Shen ${ }^{1, a}$, Jie Wang, ${ }^{1,{ }^{*}}$, Zifeng Dai, ${ }^{1, c}$ and Panzhong Lu ${ }^{1} \mathrm{~d}$ \\ ${ }^{1}$ Huazhong Agricultural University, Wuhan 430070, China

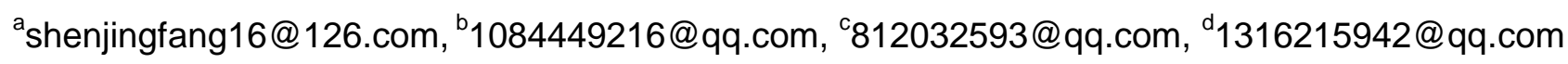 \\ *The Corresponding author
}

\begin{abstract}
Keywords: Damping model; Truncation error; Dynamic response analysis; System spectrum expansion theory; Jordan standard shape
\end{abstract}

\begin{abstract}
There were a lot of research on the error truncation analysis of the damping system. However, the theoretical methods that in the analysis of vibration structure and dynamic response are complex and diversified, and there is no completely unified theoretical standard. The main contents of this paper are to summarize the modal analysis methods of predecessors and then divide the methods into the categories from the perspective of error control. Finally, we will make effective corrections on the problem of control error terms in the damping system. In this paper, we will compare the modal superposition theory and method mentioned by the previous ones, so that we can put forward the limitations of each method which is that the analysis method involves only the complex modal solution technique of the general damping system. Therefore it is necessary to study the high efficiency of the damping system (Eigenvalues and eigenvectors). For the complex modal solution of complex damping system, the predecessors use the matrix diagonalization method generally, but there are many restrictions in the diagonalization of the matrix, therefore we can use Jordan Standard-shaped research to start a more general practice and theoretical methods In the case of the more general diagonal solution. Finally, we will prove that the improved method has some restriction on the cut off of control error by numerical simulation.
\end{abstract}

\section{Introduction}

With the continuous development of science and technology, some new composite materials was born. These new composite materials are widely used in aviation, aerospace, machine tools, automobiles and shipbuilding fields, and vibration damping has become the primary issues to be considered in the application. With the requirement of people describing the suitability and accuracy increasingly, damping problem has become a hot topic in today's research questions.

The rapid progress of modern industry makes many products developing to bigger, faster, lighter, safer and more reliable, therefore damping vibration analysis plays a more and more important role in real life. And as we all know, vibration problems are prevalent in engineering applications, such as mechanical equipment, aerospace, automobile and bridge, construction technology, etc. Due to vibration, it can generate some negative factors such as energy loss, which causes: vibration loss of mechanical facilities, unstable vibration of bridge structures and some adverse effects, this makes the relevant theories about research on how to make use of damping and existing damping model to solve the problem of vibration improvement and control has been widely researched and draw attention by professional scholars. With the development of vibration theory and its related fields. It has been changed just rely on the static strength theory for structural design concept. Because of that many structures are in motion with the external incentives or under its own power, which the main ingredients are vibration, such as vibrations in rotating machinery, aircraft flutter and vibration of vehicles, ships and other means of transport, and so on. Dynamic characteristics must be considered in these mechanical design, evaluation, which makes some problem seems to be static. But the influence of dynamic factors must be considered when doing the structure design, which makes the damping problem becomes more complicated and diversity. And it means that the complication of damping issues a bigger challenge to the research of academia and industry. A large number of facts indicate, 
vibration characteristics analysis of damping play a vitally important role in structure design and evaluation. The job that solving the problem of how to extract useful information from the result and feedback to the product design and development is full of challenging although computer technology supply a material basis to establish a more detailed model. Due to the excessively detailed model not only can increase the computational cost but also make the process of how to make correct judgment more difficult in the engineering analysis. Therefore making reasonable simplification which is based on the theory and experience has realistic significance.

Academy and industrial have published a number of documentations and reports on the damping and application since 1877[1]. This shows that although the damping is an old problem, damping analysis is a difficult problem in the mechanical structure dynamic analysis. With the rapid development of modern industry, damping is still a hot issue with great vitality. Many people have done further research and application with the problem of damping and published a lot of research literature on the damping. However, these articles tend to aim at different fields and different applications which study mainly on different theories and applications. But the theoretical method is more complex and diverse in a wide variety of vibrational structures and dynamic response analysis, that is to say there is no complete unified. Therefore this paper summarizes theoretical methods and classify systematically, and this work is a very significant research topic on further research methods of damping analysis.

There are many articles on the study of the joint, and they have made in-depth study and may progress from view of different point. And it is well known that there is damping in the mechanical system as long as there is vibration. Damping is an important characteristic parameter used to specifically represent the energy dissipation characteristics in a vibration system. Energy consumption refers to the performance of mechanical energy that can be dissipated into heat energy during the vibration process, and the process is irreversible. In the complex vibration mechanism, it is not possible to achieve the damping of the theoretical research system and accurate unity for the different damping system has different physical mechanism and its vibration principle. However, the scientific community has a large number of damping hypothesis model after the predecessors of the damping problem at continuous exploration and research. Such as viscous damping, structural damping which is widely used with a macroscopic approximation damping model. The purpose of this damping model is mainly to focus on the theoretical simplification of the calculation. Another damping model, which is widely studied and applied, is called a damping model taking into account the physical mechanism. Unlike the macro approximation model proposed by the appeal, the model studies the damping problem in a microscopic view. Mainly focus on the physical energy dissipation and other physical mechanisms, such as friction damping, fluid damping and so on.

Recently, the biggest problem is the how to solve the damping in the field of engineering impact. In engineering applications, there are proportional approximations (main diagonal matrix criteria) and frequency domain response (Hasselman criteria) for simplified methods commonly used in viscous damping systems.

Proportional approximation is most commonly simplified method used in dynamic analysis in engineering applications. The main idea is: ignore the off-diagonal elements of viscous damping matrix in modal, so as to achieve modal analysis method can be applied to non-classical damping system.

From the above description, we can gain quadratic approximation by means of approximation solution, there are two main aspects for the error. One is that in the damping models, we replaced the error that energy dissipation generated in whole system with viscous damping energy; the other is in the course of application of numerical approximation, ignored the error arising from the non-diagonal elements inthe modal viscous damping matrix errors.

Many scholars try to solve the error problem of Proportional approximation by research, which mean make calculate of the acceptable error range through proportional approximation. Thus, Hasselman proposed frequency response method in 1976. 
The two major criteria have been widely used in academic research and discussion, but with the two major criteria used in the practical application, many scholars have discovered the sufficient condition which the two major criteria are no longer ignored the off-diagonal elements of the matrix in viscous damping system, even get contrary results compared to the conclusions.

In the harmonic response analysis of engineering problems, if we considered local damping (such as car bumpers) and local viscous damping that caused by the complex mechanisms-material damping and joints part friction, most of the damping model which corresponds to damping matrix cannot meet the criteria of the proportional damping (Generally the damping matrix is not symmetric, that is mode vector cannot be diagonalized). Therefore, in the modal frequency response analysis, if we use modal analysis to solve structure frequency response, the equation converted to modal coordinates in the computational process can't be decoupled. Generally speaking, structural system under dynamic excitation, high order mode has smaller contribution, we just discussed the low order mode feature of system. In the large scale engineering problems, the calculated amount of all modes is very large, due to the effect of numerical round-off errors, high order modes are often can't get up to the required accuracy. The mainly restriction that decide structure response is low-frequency modes. hence, there will be a greater modal truncation error in harmonic response analysis. If what we get is only first $\mathrm{L}$ low-order modes, when using the first L low-order modes in frequency response function matrix and dynamic displacement vectors, we can obtain:

$$
\left\{\begin{array}{c}
H(\omega) \approx H_{M D M}(\omega)+H_{H}(\omega) \\
X(\omega) \approx H_{M D M}(\omega) F(\omega)
\end{array}\right.
$$

In which

$$
\begin{aligned}
& H_{M D M}(\omega)=\sum_{j=1}^{L} \frac{\varphi_{j} \psi_{j}{ }^{T}}{\theta_{\mathrm{j}}\left(i \omega-\lambda_{j}\right)} \\
& H_{H}(\omega)=\sum_{j=L+1}^{J} \frac{\varphi_{j} \psi_{j}^{T}}{\theta_{j}\left(i \omega-\lambda_{j}\right)}
\end{aligned}
$$

Formula(1)is the expression of modal displacement method, $H_{\mathrm{H}}$ called modal truncation error.it is a great practical significance for accurate solution of dynamic response that study how decrease or eliminate modal truncation.

\section{Classical Damping Model}

Viscous Damping Model. Viscous damping happened in the process of vibration which deformation happened in the body, when vibration occurred, some rational energy loss in the resistance of its environment, such as rational objects subjected to air or water resistance, and the energy was converted to heat energy. That is to say if the magnitude of the damping force is proportional to the instantaneous velocity of the mobile particle, in the opposite directions, recorded as:

$$
f_{D}(t)=-c \dot{x}(t)
$$

In the above formula, $f_{D}(t)$ is damping force, $c$ is viscous damping coefficient, $x(t)$ is the instantaneous velocity of the particle. The corresponding differential equations of motion is: $M \ddot{x}(t)+c \dot{x}(t)+k x(t)=f(t)$

In the above formula, $\mathrm{M}$ is mass, $\ddot{x}(t)$ is instantaneous acceleration, $x(t)$ is displacement, $f(t)$ is extra incentive loading.

Viscous damping force is proportional to the instantaneous velocity, the dynamic equations is linear equations, this feature made a great convenience in the theoretical analysis. This simplicity makes the viscous damping model became a linear damping model in mechanical vibration systems and engineering applications.

For the different degrees of freedom, recognition method of viscous damping models are not the same. For a single degree of freedom system, there are damping identification methods: recognition 
method based on energy dissipation based on time-domain response, recognition method based on frequency response. For a multiple degree of freedom system, damping identification methods are: modal analysis, response analysis method in time domain and frequency domain response method.

Structural Damping Model. In a real rational system, apart from the viscous damping, there also is structural damping. When is subjected to alternating strain force, it is a kind of capacity of dissipation that friction of materials. In theory, we assumes that the solid material damping of stress is proportional to elastic stress, when it occurs simple harmonic motion, the damping force is proportional to elastic stress and has the same direction as velocity, recorded as:

$$
f_{D}(t)=-i \eta k x(t)
$$

$i$ is imaginary number symbol, $\eta$ is damage factor of structural damping and it is a constant, $x(t)$ is displacement.

Dynamic response equation of single degree of freedom structure damping system can be written as

$m \ddot{x}(t)+k(1+i \eta) x(t)=F \exp (i \omega t)$

there is no convergence for the free dynamic response. Thus it is limited to the harmonic response in logically, when analyze the instantaneous dynamic response, it abnormal causal relationship will appear in the dynamic response.

When the frequency response function meet the condition of conjugation, we can obtain the impulse response function of a real number, the frequency domain expression of structural damping force is:

$$
F_{D}(\omega)=\mathrm{i} \eta \operatorname{sign}(\omega) X(\omega)
$$

But because of the structural damping time domain equation is ill conditions equation, and the expression of frequency domain equation is various, in the concise various cases, made the structural damping bear large controversy in theoretical study and engineering application. Structural damping loss factor is a commonly used indicator of evaluating damping. Defined as a one-week period the dissipation of energy and the ratio of the maximum capacity of the cycle. Viscous damping in the system loss factor, damping loss factor in the system is constant.

This character determinate the energy loss of viscous damping increasing along with frequency increase one period; structural damping not only concise but also can fit well in most energy dissipation test for solid materials, consequently widely used in structural dynamics problems.

Fractional Damping Model. Friction damping belonging to the damping which related to the physical mechanism, the corresponding damping model correspond with the energy dissipation of friction, can be more precise handling physics problem the in the friction.

Friction damping model can describe the sliding interface friction damping under normal pressure. Assuming that the damping force is proportional to the normal force, regardless of the velocity, direction and movement are in the opposite direction, recorded as:

$$
\begin{aligned}
& f_{D}(t)=\operatorname{sign}(\dot{x}(t)) f_{\mu} \\
& f_{\mu}=\mu f_{N}
\end{aligned}
$$

In the above formula, $f_{\mu}$ is friction force, $\mu$ is friction coefficient, $f_{N}$ is normal pressure.

Fluid Damping Model. Fluid damping damping model is often used to analyze fluid dynamics problems, which are widely used in Aerospace Dynamics. There are some common damping: liquid viscous damping, dynamic damping of air. It can be recorded as:

$$
f_{D}(t)=\operatorname{sign}(\dot{x}(t)) \alpha \dot{x}(t)^{2}
$$

More general form is:

$$
f_{D}(t)=\beta \dot{x}(t)|\dot{x}(t)|^{n-1}
$$

In the above formula: $\alpha$ and $\beta$ are damping constant coefficient, $\operatorname{sign}(t)$ is sign function. 


\section{Theory Analysis and Method of Damping System}

To sum up, Damping assumption can be roughly divided into two class, namely macro approximate model (for example: viscous damping models, structural damping model) and the damping model that involved physical mechanism(for example: friction damping models, fluid damping damping, interface damping models, etc). For years, scholars have done a lot of research on the dynamic analysis of damping model, consequently form a more perfect system. Next, scholars will study the dynamic analysis of damping system theory and method in detail.

Dynamic Analysis Theory. Damping exists in any practical application of variety engineering systems, and its physical mechanism is very cumbersome. Although there are many relative discuss and in-depth study for different physical mechanism, in a variety of complex and multiple system mechanism, damping is often a complex combinations of various damping models, this makes a great challenge for the calculation of damping in the process of dynamic analysis. To simplified dynamic analysis, many scholars have to replace the whole vibration system with viscous damping energy dissipation.

Setting damping system has been divided into multiple degree of freedom system, recorded as: $M \ddot{x}(t)+C \dot{x}(t)+K x(t)=f(t)$

In the above formula, $M C$ and $K$ stand for mass matrix, viscous damping matrix and stiffness matrix respectively. $f(t)$ and $x(t)$ are exciting force vector and displacement vector. usually, system matrix is real symmetric matrix, mass matrix $M$ is positive definite matrix, damping matrix $C$ and stiffness matrix $K$ are non-negative definite at least.

Setting $U$ is not damped system modal matrix(real modal matrix),

$\widetilde{C}=U^{T} C U$

If $\tilde{C}$ is a diagonal matrix, we can decoupling the damping system to be a single degree of freedom equation through real-mode, so as to reach the propose of simplified calculation. Eq. 1 is called a damped system (proportional damping system).

Assuming that the damping matrix $C$ can be expressed as a linear combination of the mass matrix $M$ and the stiffness matrix $K$, that is to say:

$C=\mu_{m} M+\mu_{k} K$

$\mu_{m}$ and $\mu_{k}$ are positive constant, this damping is the special form of this classic damping [1]. In theory, determine the equivalent conditions of judging classical damped system in viscous damping system is:

$C M^{-1} K=K M^{-1} C$

For classical damping system, Li [2] defines a non-classical damping system: If the system cannot decoupling through the real-mode (for example, for viscous damping system, modal viscous damping matrix is non-diagonal matrix), hence the system is non-classical damping system (non-proportional damping system).

State-Space Method. The state space method is an important method in the non classical damping system. The state space equation of viscous damping system Eq.1 is expressed as:

$\dot{z}(t)=A z(t)+r(t)$

In the above formula

$$
A=\left[\begin{array}{cc}
0 & I \\
-M^{-1} K & -M^{-1} C
\end{array}\right], z(t)=\left\{\begin{array}{l}
x(t) \\
\dot{x}(t)
\end{array}\right\} r(t)=\left\{\begin{array}{c}
0 \\
-M^{-1} f(t)
\end{array}\right\}
$$

$I$ is a unit matrix, its dimension is the same as that of the system matrix, Matrix $A$ is not symmetric matrix. So it need left and right characteristic equation of state Eq.3 to receive left, right modal vector to construct a double modal orthogonality relation.

through state equation he state equation of two order linear system Eq.1 is converted to a linear system, which can transform viscous damping system into he first-order linear system which is similar to without damping system, therefore, it can be extended to the non classical damping system, which belongs to the non damping system. 
In 1958, Foss[3] proposed a new state space equation.

$\left[\begin{array}{cc}C & M \\ M & 0\end{array}\right]\left\{\begin{array}{l}\dot{x}(t) \\ \ddot{x}(t)\end{array}\right\}+\left[\begin{array}{cc}K & 0 \\ 0 & -M\end{array}\right]\left\{\begin{array}{c}x(t) \\ \dot{x}(t)\end{array}\right\}=\left\{\begin{array}{c}f(t) \\ o\end{array}\right\}$

The biggest advantage of this method is that the system matrix is symmetrical, but the disadvantage is that it increases the system dimension, which increases the storage capacity and reduces the computation efficiency and time.

Modal Acceleration Method. Modal acceleration method (MAM) [4] is the most commonly used of the modal approximation method, which is a kind of calculating frequency response function of a modal acceleration method based on the modal expansion method, power series expansion principle and frequency shift technique.

Let us consider a discrete structure, $\mathrm{n}$ degrees of freedom equation can be expressed as a form: $M \cdot \ddot{u}(t)+C \cdot \dot{u}(t)+K \cdot u(t)=F \cdot w(t)$

$u(t)=\left\{u_{1}(t) \cdots u_{n}(t)\right\}, w(t)=\left\{w_{1}(t) \cdots w_{l}(t)\right\}, M, C$ and $K$ represent n-dimensional matrix mass, viscous damping and elastic stiffness. In order to reduce the existence of error problems in large-scale structural systems, the equations of motion are usually defined in modal spaces. So the real values can be defined by equation

$$
\tilde{\omega}_{\mathrm{j}} M \cdot \tilde{\phi}=K \cdot \tilde{\phi}_{j}
$$

When the external excitation frequency is in the low frequency region system, the method can be used to cut off and accelerate the system's low and high order modes concurrently. It makes the modal number required for the modal expansion to be greatly reduced. The two-dimensional frame structure calculation shows that effect of modal acceleration is obvious, usually only a few items included in the former of the power series can make the frequency response function to achieve high precision.

Modal Strain Energy Method. Modal strain energy method belongs to the dynamic analysis method which is easy to implement, and it is a kind of approximate method proposed by Mead, the basic idea is that the ratio of the damper and the maximum strain energy per cycle system can determine the equivalent damping ratio, and estimates approximately damping effect of structure.

1. The basic assumptions of the MSE method are as follows. The full damping of the structure is the classical damping, the meaning is that the response of the real mode is decoupled;

2. Modal damping is viscous, and the memory effect of dampers is completely neglected in the analysis.

The analysis of the first hypothesis is satisfied; the second hypothesis will make the analysis result inaccurate. Because the MSE method is a kind of forced decoupling method, so it will cause some errors in the analysis. MSE method has been widely used in the dynamic analysis of viscoelastic damping system by the development of Johnson [5] and others.

Direct Frequency Domain Method. In modal response analysis, the modal method variables are less, the solution speed is quick, it is suitable for the bigger model, usually chooses a certain range of modal frequency to calculate and its accuracy is lower than direct method, but it is often beneficial to deal with the problem of response reduction model dynamic degrees of freedom. Unfortunately, in the process of reduction, the change of the mode will have a negative effect on the accuracy of the calculation. In order to solve the problem of direct acquisition of frequency response, the direct frequency domain method is widely concerned.

Mode Superposition Method. According to the direct frequency domain method, the modal superposition method and the high accuracy of modal superposition method has improved to some extent, this method improves the accuracy of the FRF matrix and dynamic response speed.

Modal superposition method is that each order modal coordinate transformation using modal analysis to obtain was carried out on the vibration response equation and the equation decoupling, under the physical coordinate system, the solution of multiple degree of freedom system turns into Multiple solution of the single degree of freedom system under Modal coordinate system, the response of each mode is obtained, and the response of the system under the real physical coordinate system is obtained. 


\section{The Limitations of Classic Methods}

The state space method doubles the system dimension in the application process, thereby increasing the storage capacity and reducing the efficiency and time of the calculation.

The modal acceleration method is an approximation, which only considers the contribution of the high order mode to the static displacement. In the actual engineering, the external excitation frequency is often higher and is in the middle frequency region of the system. In this case, Acceleration method, you need to know all the medium and low order modes of the system. This is undoubtedly inconvenience to the analysis of the characteristics of the system, while ignoring the contribution of speed and acceleration of the dynamic response, but also makes it in the lower frequency range of the greater error, so its accuracy needs to be further improved.

Modal strain energy method has the advantage of being able to efficiently analyze medium and low damping materials, but for high precision damping materials can not be precisely processed, and can not do direct frequency response analysis.

For the direct frequency domain method, the biggest advantage is that the experimental data can be directly obtained by the storage modulus and dissipation modulus, which can be analyzed on the damping system for finite element modeling. However, the limitation lies in the fact that the analysis is limited to the frequency domain problem, and it is necessary to constantly update the damping matrix of each frequency point in the analysis process. The computational complexity is very large for large-scale engineering problems.

Dynamic analysis based on time domain equation is a new method to study the dynamic performance of damping system. In terms of dynamic analysis, it is very convenient and economical. In terms of frequency domain response analysis, the method is superior to the direct frequency domain method when dealing with large-scale practical problems.

The advantage of modal superposition method is that the modal superposition can not only obtain the response value of the low order mode of the system, but also the lower order modal response information can be used to obtain the response approximation of the high order mode of the system. Compared with the practical improved modal superposition algorithm, the applicable load of the algorithm can be extended to the ordinary load (such as the sudden load). And make up for the shortcomings of finite element analysis to ignore the high-level information, but also can be applied to the use of dynamic sub-structure to solve the modal information of large dynamic system response. And its shortcomings lies in the analysis of the damping system, only consider the diagonal elements of the matrix and ignore the non-diagonal elements on the modal truncation error, so that the numerical calculation of large errors, but also in the posteriori error estimation The process is rather cumbersome.

\section{Improvement of Dynamic Response Method}

The limitations of the above mentioned modal superposition theory and methods lie in the analysis of the complex modes of the general damping system, So it is necessary to study the efficient (eigenvalue and eigenvector)solving method for the general damping system. As we all know, diagonal matrix can be considered the most simple one of the matrix, so it is widely used in practical engineering problems. Now we examine a linear transformation of the matrix which can be diagonal matrix in a group of appropriate base. Now we introduce the Jordan (Jordan) standard.

The form is

$$
J=\left[\begin{array}{cccccc}
\lambda & 0 & \cdots & 0 & 0 & 0 \\
1 & \lambda & \cdots & 0 & 0 & 0 \\
\vdots & \vdots & & \vdots & \vdots & \vdots \\
0 & 0 & \cdots & 1 & \lambda & 0 \\
0 & 0 & \cdots & 0 & 1 & \lambda
\end{array}\right]_{t \times t}
$$


This form of matrix is called Jordan standard block, and $\lambda$ is plural. Quasi diagonal matrix is composed of a plurality of Jordan block, and the quasi diagonal matrix is called Jordan matrix, its general form is:

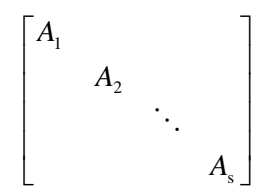

In which

$$
A_{i}=\left[\begin{array}{ccccc}
\lambda_{i} & & & & \\
1 & \lambda_{i} & & & \\
& 1 & \ddots & & \\
& & \ddots & \lambda_{i} & \\
& & & 1 & \lambda_{i}
\end{array}\right]_{k_{i} \times k_{i}}
$$

It is possible that there are some equal during $\lambda_{\mathrm{i}}$.

Any complex field matrix can be diagonalized. But the analyzed elements do not necessarily belong to the complex field in the actual damping matrix analysis. And as we all know, any matrix can be calculated by a series of Jordan standard, which can achieve the purpose of simplifying the calculation and make damping analysis become more extensive attention in the application. Because of this limitation, the analysis of the actual damping matrix will be more generalized, so it is very practical to use this theory to improve the dynamic response method.

\section{Conclusion}

On the problem of reducing or eliminating the modal truncation error, although there are a lot of research on a system without damping and viscous damping system, most of the methods and theories mentioned in the previous studies only relate to the complex modal solution technique for general damped systems, there is little literature on the modal truncation problems of viscoelastic damping system are discussed. So it is necessary for the non completeness of damping system to conduct the efficient solution and reduce the modal truncation method. However, some of the common methods mentioned above only relate to the complex modal solution technique for general damped systems, so the research on the general damping system is a new Solving method in terms of innovation and development. Generalized modal superposition form in the application of dynamic response analysis of other problems remains to be further studied and discussed, such as damping identification problems, sensitivity analysis, the dynamic change and dynamic optimization problems.

\section{Acknowledgments}

This work was supported by university students science and technology innovation project (2017BC027) and Higher school university mathematics teaching research and development center project (CMC20160408).

\section{References}

[1] Rayleigh, L. Theory of sound (two volumes). New York: Dover Publications, 1877.

[2] LiL, HuYJ and Wang XL (2013a) Design sensitivity analysis of dynamic response of nonviscously damped systems. Mechanical Systems and Signal Processing 41: 613-638.

[3] Foss, K. A. Coordinates which uncouple the equations of motion of damped linear dynamic systems. Journal of Applied Mechanics-ASME, 1958, 1958 (25): 361-364.

[4] Structural Dynamic Analysis with Generalized Damping Models Analysis.

[5] Palmed A, Ricciardelli F, Muscolino G De Luca A. Random vibration of system the viscoelastic memory [J].Journal of Engineering Mechanics,2004,130(9):1052—1061. 